\title{
Improving the Accuracy of Soil Moisture Retrievals Using the Phase Difference of the Dual-Polarization GNSS-R Interference Patterns
}

\author{
A. Alonso-Arroyo, Student Member, IEEE, A. Camps, Fellow, IEEE, A. Aguasca, Member, IEEE, \\ G. Forte, Student Member, IEEE, A. Monerris, Member, IEEE, C. Rüdiger, Member, IEEE, \\ J. P. Walker, Member, IEEE, H. Park, Member, IEEE, D. Pascual, Student Member, IEEE, and \\ R. Onrubia, Student Member, IEEE
}

\begin{abstract}
Soil moisture (SM) is a key parameter in the climate studies at a global scale and a very important parameter in applications such as precision agriculture at a local scale. The Global Navigation Satellite Systems Interference Pattern Technique (IPT) has proven to be a useful technique for the determination of SM, based on observations at vertical polarization (V-Pol) due to the Brewster angle. The IPT can be applied at both V-Pol and horizontal polarization (H-Pol) at the same time, observing the Brewster angle only at V-Pol. This letter presents a measurement technique based on tracking the phase difference between V-Pol and H-Pol interference patterns to improve the accuracy of the Brewster angle determination and, consequently, that of the SM retrievals. This technique benefits from the different phase behavior of the reflection coefficients between $\mathrm{H}-\mathrm{Pol}$ and V-Pol in the angular observation range. To be sensitive to the phase difference, the Rayleigh criterion for smooth surfaces must be accomplished. This technique is not sensitive to topography as it is intrinsically corrected. Experimental results are presented to validate the proposed algorithm.
\end{abstract}

Index Terms-Global Navigation Satellite Systems (GNSS), GNSS reflectometry (GNSS-R), Interference Pattern Technique (IPT), soil moisture (SM).

\section{INTRODUCTION}

$\mathbf{T}$ HE measurement of soil moisture (SM) at global scale has been one of the holy grails in remote sensing during the last decades. Knowing the evolution of SM at a global scale can help to study the water cycle and the climate. At a more local scale, the knowledge of SM is quite important in fields such as precision agriculture and water management, in order to maximize the productivity by optimizing the use of water. In the microwave remote sensing field, active and

Manuscript received December 30, 2013; revised February 28, 2014 and April 8, 2014; accepted April 17, 2014. This work was supported by the Spanish Ministry of Science and Innovation project, "AROSA-Advanced Radio Ocultations and Scatterometry Applications using GNSS and other opportunity signals" under Grant AYA2011-29183-C02-01/ESP.

A. Alonso-Arroyo, A. Camps, A. Aguasca, G. Forte, H. Park, D. Pascual, and R. Onrubia are with the Department of Signal Theory and Communications, Universitat Politècnica de Catalunya BarcelonaTech, 08034 Barcelona, Spain (e-mail: alberto.alonso.arroyo@tsc.upc.edu).

A. Monerris, C. Rüdiger, and J. P. Walker are with the Department of Civil Engineering, Monash University, Clayton, Vic. 3168, Australia.

Color versions of one or more of the figures in this paper are available online at http://ieeexplore.ieee.org.

Digital Object Identifier 10.1109/LGRS.2014.2320052 passive techniques have been used to determine SM, such as the synthetic aperture radar [1], [2], microwave radiometers (e.g., SMOS and AQUARIUS missions [3]-[5]), and, more recently, the Global Navigation Satellite Systems (GNSS) reflectometry [6], which is the basis of the technique proposed in this letter.

GNSS reflectometry (GNSS-R) started in 1991, when the Global Positioning System (GPS) receiver of a French military aircraft got accidentally locked to the GPS reflected signal instead of the direct one, giving an erroneous navigation solution [7]. This incident showed the feasibility of sensing the GNSS reflected signals over the ocean. In 1993, the European Space Agency proposed the PAssive Reflectometry and Interferometry System concept [8], a multistatic passive technique for retrieving mesoscale ocean altimetry. Since then, several other uses of GNSS opportunity signals have appeared (e.g., [9]).

The sensitivity of GNSS-R techniques to SM has been proved through theoretical studies and field campaigns over the past ten years. Several techniques from different platforms, i.e., airborne and ground based, have been used assessing their performance. The first approach was based on measuring the power difference of direct and reflected GNSS signals, which is the terrain reflectivity. From the reflectivity values, the dielectric constant is first inferred and then the SM. In 2003, dualpolarization observations, i.e., horizontal (H-Pol) and vertical (V-Pol), were proposed to be used in order to reduce the impact of the attenuation in the reflected signals due to surface roughness [10]. Theoretically, surface roughness should equally affect both polarizations, but experimental data showed that it was not performing as expected [10]. In 2004, an airborne field experiment was conducted with a nadir-looking left-hand circular polarization (LHCP) GPS antenna showing a difference in the reflected LHCP power before and after a rain event. This stated that reflectivity measurements using LHCP polarization had sensitivity to SM from airborne platforms [11]. In 1998, the use of GNSS multipath was proposed for measuring the ground complex permittivity [12]. Since 2007, multipath information has been used to retrieve different geophysical parameters, including SM, using right-hand circular polarization (RHCP) GPS geodetic antennas [13]-[15]. In 2009, the observation of the Brewster angle, i.e., $\theta_{B}$, on the V-Pol Interference Pattern Technique (IPT) was proposed to retrieve the SM in ground-based applications [16]. This concept was extended in 2011 to vegetation scenarios looking to the different minimum 
amplitude oscillations due to presence of a vegetation layer [17]. Finally, direct and reflected in both LHCP and Right Hand Circular Polarization (RHCP) have been used to retrieve SM. The technique used is based on the Interferometric Complex Field (ICF), and inferring the reflection coefficient by dividing the averaged waveforms for direct and reflected GNSS signals [18].

This letter is divided in four sections, beginning with an introduction. Section II presents the methodology used for the SM retrieval with some theoretical simulations. Section III details the instrument used for the experimental field campaign and presents the results obtained. Finally, a discussion about the methodology used and the conclusions of this letter are presented in Section IV.

\section{Dual-Polarization IPT: Theoretical Analysis}

The dual-polarization IPT is an evolution of the IPT [16], [17], based on combining the information provided by the interference patterns at H-Pol and V-Pol. The IPT is based on the analysis of the coherent electromagnetic (EM) addition of the direct and reflected signals (fading) using a linearly polarized antenna looking to the horizon, a fact also known as one-ray multipath. This creates an interference pattern with a high-frequency component related to the multipath and a low-frequency component related to the antenna pattern. The theoretical model is presented as

$$
P_{R} \propto F_{n}(\theta) \cdot\left|E_{0}\right|^{2} \cdot|1+| R_{q}\left(\theta, \varepsilon_{r}\right)\left|e^{j\left(\Delta \phi+\phi_{R_{q}}\left(\theta, \varepsilon_{r}\right)\right)}\right|^{2}
$$

where $P_{R}$ is the received power, $F_{n}(\theta)$ is the antenna radiation pattern assumed to have symmetry of rotation, $E_{0}$ is the incident electric field amplitude, $\left|R_{q}\left(\theta, \varepsilon_{r}\right)\right|$ is the absolute value of the Fresnel reflection coefficient at $q$ polarization, $\phi_{R_{q}}\left(\theta, \varepsilon_{r}\right)$ is the phase of the reflection coefficient at $q$ polarization, $\Delta \phi=$ $(4 \pi / \lambda) h \sin (\theta)$ is the phase difference due to the different electrical paths between direct and scattered EM waves, $\lambda$ is the wavelength ( $\sim 19 \mathrm{~cm}$ at $\left.f_{L 1}=1575.42 \mathrm{GHz}\right), h$ is the height of the receiving antenna, $\varepsilon_{r}$ is the soil surface dielectric constant, and $\theta$ is the elevation angle of the GNSS satellites.

The conventional IPT uses V-Pol to retrieve the $\theta_{B}$ position, which produces a minimum amplitude point in the interference pattern that varies its angular position as a function of SM. From the Brewster angle, the dielectric constant $\left(\varepsilon_{r}\right)$ of the terrain can be inferred, which can be associated to a SM value [16], [17].

In (1), there are two terms that depend on polarization $q$ : $\left|R_{q}\left(\theta, \varepsilon_{r}\right)\right|$, which is related to the amplitude of the highfrequency oscillations, and $\phi_{R_{q}}\left(\theta, \varepsilon_{r}\right)$, which is related to the angular positions of the maxima and minima points of this highfrequency oscillation. The maxima points have $1+\left|R_{q}\left(\theta, \varepsilon_{r}\right)\right|$ amplitude and occur when $\Delta \phi+\phi_{R_{q}}\left(\theta, \varepsilon_{r}\right)$ is an even multiple of $\pi,(2 n \pi)$. The minima points have $1-\left|R_{q}\left(\theta, \varepsilon_{r}\right)\right|$ amplitude and occur when $\Delta \phi+\phi_{R_{q}}\left(\theta, \varepsilon_{r}\right)$ is an odd multiple of $\pi$, $((2 n+1) \pi)$. Hence, the positions of the maxima and minima have two contributors: $\Delta \phi$, which is independent from the polarization, and $\phi_{R_{q}}\left(\theta, \varepsilon_{r}\right)$, which is polarization dependent; thus, it depends on the soil properties.

In order to understand how the scattering process affects the reflected wave, simulations using the Fresnel reflection coefficients have been performed for different SM values. Note that the Fresnel reflection coefficients can be used to model the reflected signal because it is considered that reflection surface accomplishes the Rayleigh criterion for smooth surfaces [19]. Consequently, the coherent component dominates against the incoherent one, and an interference pattern is seen. If the Rayleigh criterion for smooth surfaces is not accomplished, the incoherent component would dominate, the reflection coefficient phase would be random, and no interference pattern would be seen. In previous works, it was seen that the coherent component dominates against the incoherent one [17]. Then, the Fresnel reflection coefficients are given by

$$
\begin{aligned}
& r_{h_{i, i+1}} \\
& =\frac{\sqrt{\varepsilon_{r_{i}}-\varepsilon_{r_{i}} \sin \left(\theta_{\text {inc }}\right)^{2}}-\sqrt{\varepsilon_{r_{i+1}}-\varepsilon_{r_{i}} \sin \left(\theta_{\text {inc }}\right)^{2}}}{\sqrt{\varepsilon_{r_{i}}-\varepsilon_{r_{i}} \sin \left(\theta_{\text {inc }}\right)^{2}}+\sqrt{\varepsilon_{r_{i+1}}-\varepsilon_{r_{i}} \sin \left(\theta_{\text {inc }}\right)^{2}}} \\
& r_{v_{i, i+1}} \\
& =\frac{\varepsilon_{r_{i+1}} \sqrt{\varepsilon_{r_{i}}-\varepsilon_{r_{i}} \sin \left(\theta_{\text {inc }}\right)^{2}}-\varepsilon_{r_{i}} \sqrt{\varepsilon_{r_{i+1}}-\varepsilon_{r_{i}} \sin \left(\theta_{\text {inc }}\right)^{2}}}{\varepsilon_{r_{i+1}} \sqrt{\varepsilon_{r_{i}}-\varepsilon_{r_{i}} \sin \left(\theta_{\text {inc }}\right)^{2}}+\varepsilon_{r_{i}} \sqrt{\varepsilon_{r_{i+1}}-\varepsilon_{r_{i}} \sin \left(\theta_{\text {inc }}\right)^{2}}}
\end{aligned}
$$

where $r_{h}$ and $r_{v}$ are the reflection coefficients for H-Pol and V-Pol, respectively; $\varepsilon_{r_{i, i+1}}$ are the dielectric constants of layer $i$ and layer $i+1$; and $\theta_{\text {inc }}$ stands for the incidence angle.

The whole reflection coefficient is computed using a $m$-layer reflection model [16], [20] with a validated $\varepsilon_{r}$ model for the soil [21]. For the sake of simplicity, a three-layer reflection model has been used (air+two soil layers). Surface roughness acts as an attenuation on the Fresnel reflection coefficients' amplitude and does not affect the phase. Equation (4) shows the implementation of the three-layer model used [16], i.e.,

$$
R=e^{-\left(\frac{4 \pi \sigma}{\lambda}\right)^{2}} \cdot \frac{r_{q_{1,2}}+r_{q_{2,3}} e^{S} e^{j 2 \phi}}{1+r_{q_{1,2}} r_{q_{2,3}} e^{S} e^{j 2 \phi}}
$$

where $\sigma$ is the surface root-mean-square height or roughness, $S$ is the surface correction parameter, and $\phi$ is the phase term associated to the interaction between layers.

This model includes the multiple interferences coming from the transmitted and reflected waves in the interference layer, taking into account the boundary conditions of the electric and magnetic fields [20]. Fig. 1 shows the simulations of the reflectivity and the reflection coefficient phase at H-Pol and V-Pol as a function of the elevation angle. A soil composition of $78 \%$ sand and $22 \%$ clay (between sandy loam and loamy sand soil) has been used in the simulations to compute the dielectric constant. Fig. 1(a) shows the power reflectivity (amplitude squared of the reflection coefficients) at $\mathrm{H}-\mathrm{Pol}$ (diamonds) and V-Pol (squares) as a function of the elevation angle, i.e., $\theta_{\text {elev }}$, which is the complimentary of the incidence angle $\left(\theta_{\text {inc }}=\right.$ $\left.90-\theta_{\text {elev }}\right)$. The presence of the Brewster angle is seen as a minimum amplitude point (V-Pol), and its angular position varies as a function of the SM content. Note that, at H-Pol, the higher the SM content, the higher the reflectivity. Fig. 1(b) shows the phase of the simulated reflection coefficients. The $\mathrm{H}-\mathrm{Pol}$ reflection coefficient phase is very close to $180^{\circ}$; it does not vary neither with the elevation angle, nor with the SM content. The V-Pol reflection coefficient phase varies as a function of the elevation angle and the SM content. Note that, by comparing Fig. 1(a) and (b), it is shown that the Brewster 


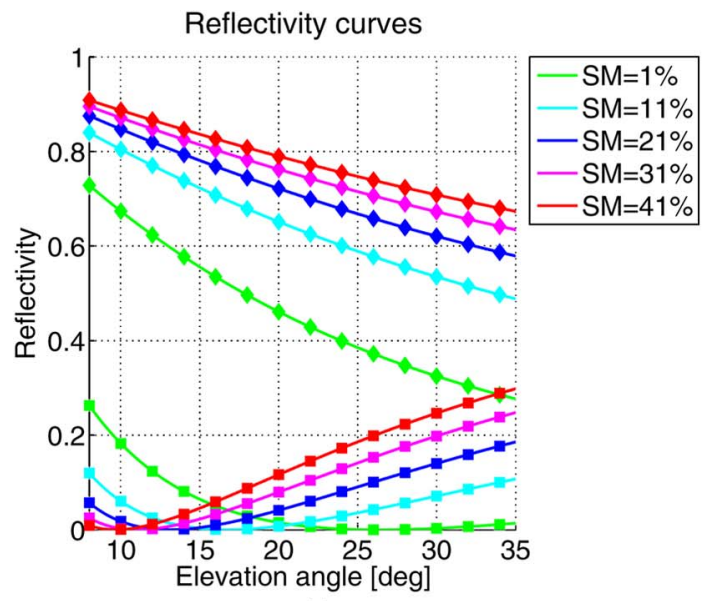

(a)

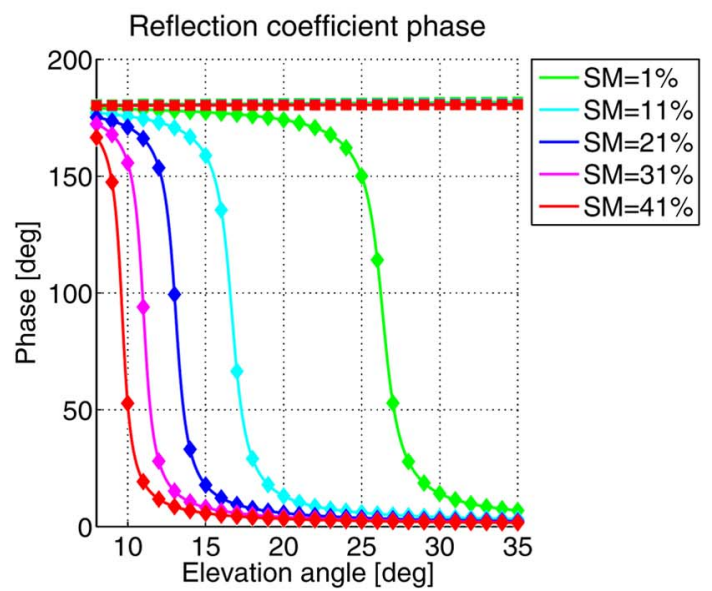

(b)

Fig. 1. Reflection coefficient curves as a function of SM: (diamonds) H-Pol; (squares) V-Pol. (a) Amplitude curves. (b) Phase curves.

angle position at V-Pol occurs when the V-Pol reflection coefficient phase is $90^{\circ}$, which means that the real part of the V-Pol reflection coefficient is very close to 0 ; thus, the imaginary part is much higher than the real part and dominates. Therefore, if the phase difference between H-Pol and V-Pol is tracked as a function of $\theta_{\text {elev }}$, the SM content can be estimated.

In order to analyze how this phase change affects the resulting interference patterns, two interference patterns for $\mathrm{SM}=$ $10 \%$ and $30 \%$ have been simulated for a 3.6-m antenna height. Fig. 2(a) and (b) shows the simulations performed. Comparing the blue dashed lines ( $\mathrm{H}-\mathrm{Pol})$ with the green lines (V-Pol), it is shown that, for low elevation angles $\left(\theta_{\text {elev }}<\theta_{B}\right)$, the H-Pol and V-Pol IPTs are in phase, whereas for $\theta_{\text {elev }}>\theta_{B}$, they are in counterphase. This is better seen just by comparing the position of the maxima and the minima as they are first coincident, whereas for $\theta_{\text {elev }}>\theta_{B}$, local maxima at H-Pol are coincident with local minima at V-Pol and vice versa. This trend is seen in both figures, changing the $\theta_{\text {elev }}$ range when they are in phase or counterphase due to the change in the $\theta_{B}$ position, which is directly related to a SM change.

Therefore, by computing the phase difference between the $\mathrm{H}-\mathrm{Pol}$ and V-Pol interference patterns, and taking into account that the phase difference due to the different path between direct and reflected GNSS signals is the same for both polarizations, the H-Pol and V-Pol reflection coefficient phase difference can

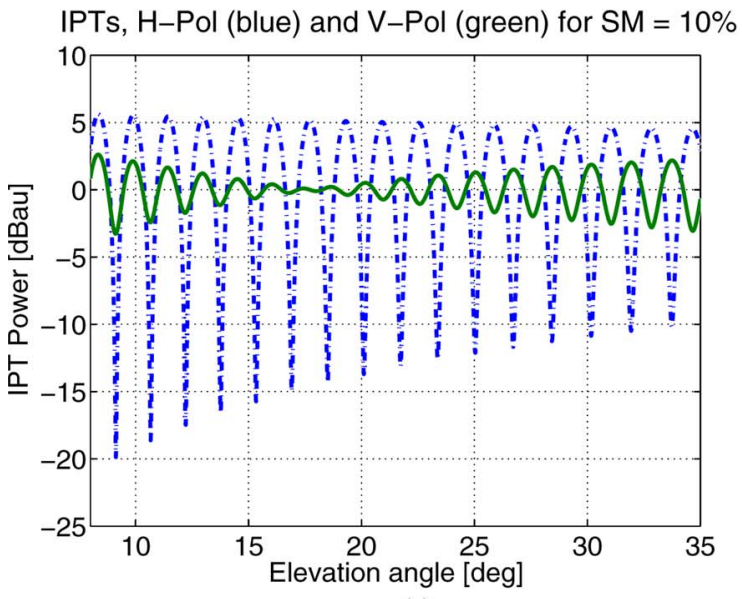

(a)

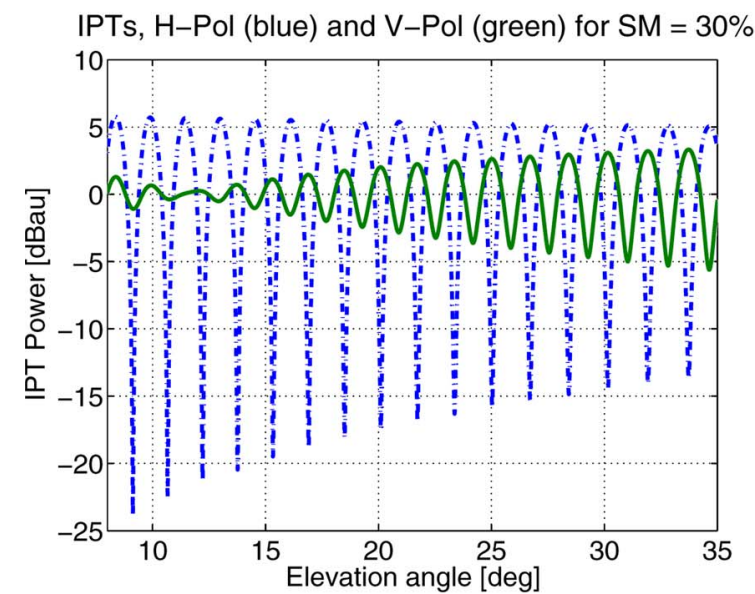

(b)

Fig. 2. Interference pattern simulated for two different SM values: $10 \%$ and $30 \%$. (Blue dashed line) H-pol. (Green line) V-Pol. (a) 10\% SM content. (b) $30 \%$ SM content.

be estimated. Then, by comparison with theoretical simulations, the SM content is retrieved.

To measure the phase difference between the H-Pol and V-Pol interference patterns, the whole phase evolution of each interference pattern must be separately retrieved. To do so, the first step is to retrieve the position of maxima and minima points. The phase difference between consecutive relative maxima and minima is $180^{\circ}$. The first relative maximum or minimum for each interference pattern is taken as a reference, and from there, the whole phase of the interference pattern can be inferred. Considering that the phase evolution between consecutive maxima and minima follows a quasi-linear phase variation, the whole interference pattern phase can be retrieved with $2 \pi$ phase jumps. After that, the phase must be unwrapped to obtain the whole phase evolution. After phase unwrapping, the phase difference between H-Pol and V-Pol is directly the difference between the phase of the reflection coefficients at each elevation angle. Fig. 3 shows the phase difference between H-Pol and V-Pol interference patterns in Fig. 2(a) and (b) as a function of $\theta_{\text {elev }}$. An oscillation in the phase retrieved curve occurs for $\theta_{\text {elev }}>\theta_{B}$ due to an uncertainty in the determination of the maxima and minima positions. However, the key point in the algorithm is the identification of the $90^{\circ}$ phase difference, which corresponds to the position of $\theta_{B}$. 


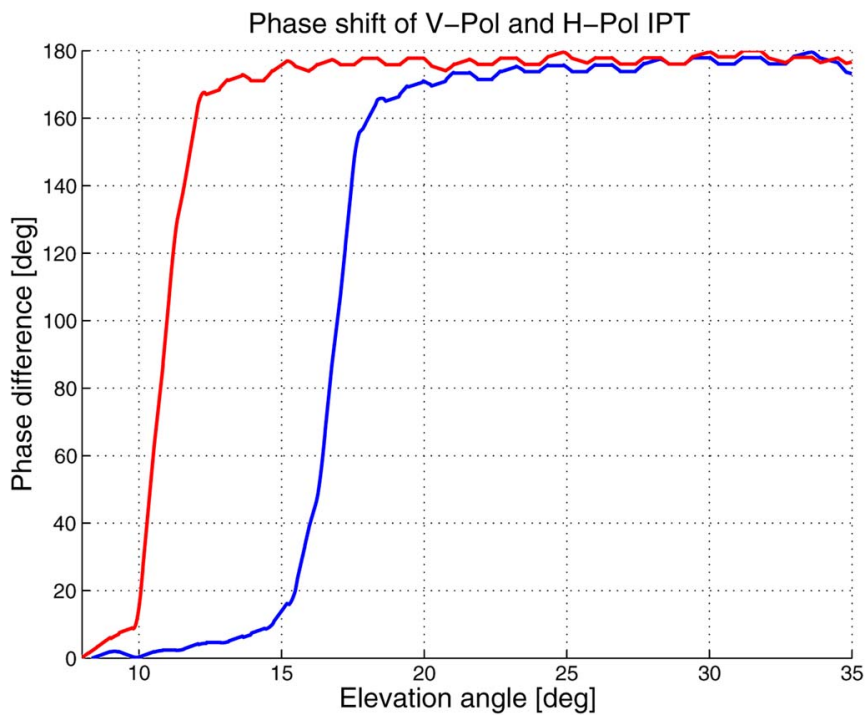

Fig. 3. Phase difference of the interference patterns simulated at H-pol and V-Pol for two different SM values: $10 \%$ (blue) and $30 \%$ (red).

A sensitivity analysis has been performed based on a series of simulations varying the surface roughness and SM conditions. The accuracy of the technique at the $90^{\circ}$ phase shift is $2.5 \%$, which is comparable with calibrated conventional SM probes [22].

\section{ANALYSIS OF EXPERIMENTAL DATA}

To experimentally test the concepts proposed in the previous section, the dual-polarization SMIGOL (PSMIGOL) has been designed as an extension of the original one [16], [17]. The original SMIGOL instrument was conceived for V-Pol only and uses the $\theta_{B}$ information. The PSMIGOL has a dual-polarization antenna ( $\mathrm{H}-\mathrm{Pol}$ and $\mathrm{V}-\mathrm{Pol})$, with a very symmetric pattern in order to have the same antenna gain for the direct and reflected GNSS signals. The cross-polar ratio of the antenna is larger than $20 \mathrm{~dB}$ to warrant negligible polarization mixing. The design of the antenna is based on the previous one with two extra feeding points for the H-Pol. After each antenna, a low noise amplifier is introduced to optimize the receivers' sensitivity. Then, each polarization is connected to its own reflectometer back end. This means that each polarization has its own separate receiving chain. To be able to store and time reference the data for each channel separately, a change in the controlling system has been applied. The data stored are finally processed by a dedicated software package.

A field experiment was conducted on July 16-31, 2013, at Yanco, New South Wales, Australia, (see Fig. 4). The observation field is a grassland, where the grass layer height was lower than $5 \mathrm{~cm}$ during the measurement period. A PSMIGOL instrument was installed on July 16, 2013 on a 3.5-m wooden mast to avoid undesired reflections (see Fig. 4). The green wooden fence was installed for security reasons (cows), selecting materials that minimize reflections without distorting the shape of the interference patterns. Its shape is close to an equilateral triangle of $3 \mathrm{~m}$ side and a height of $1.5 \mathrm{~m}$. This structure allows acquiring interference patterns free of undesired reflections along the useful elevation angle range, which is between $5^{\circ}$ and $45^{\circ}$ due to antenna pattern beamwidth.

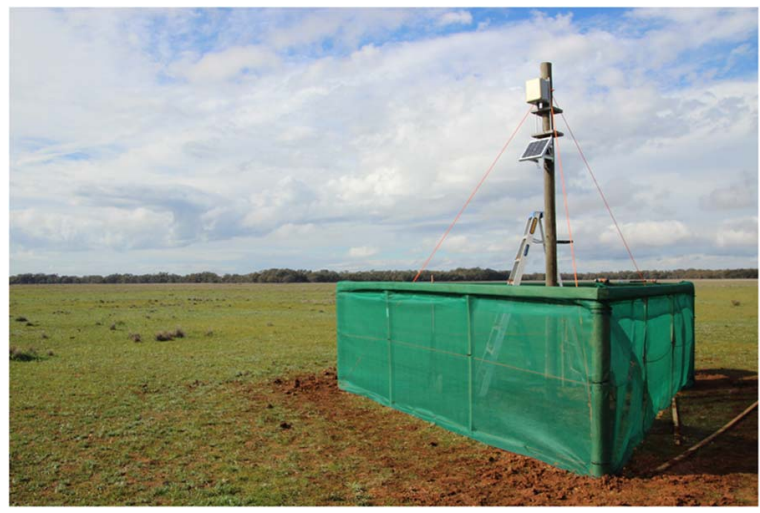

Fig. 4. Observation field and PSMIGOL setup.

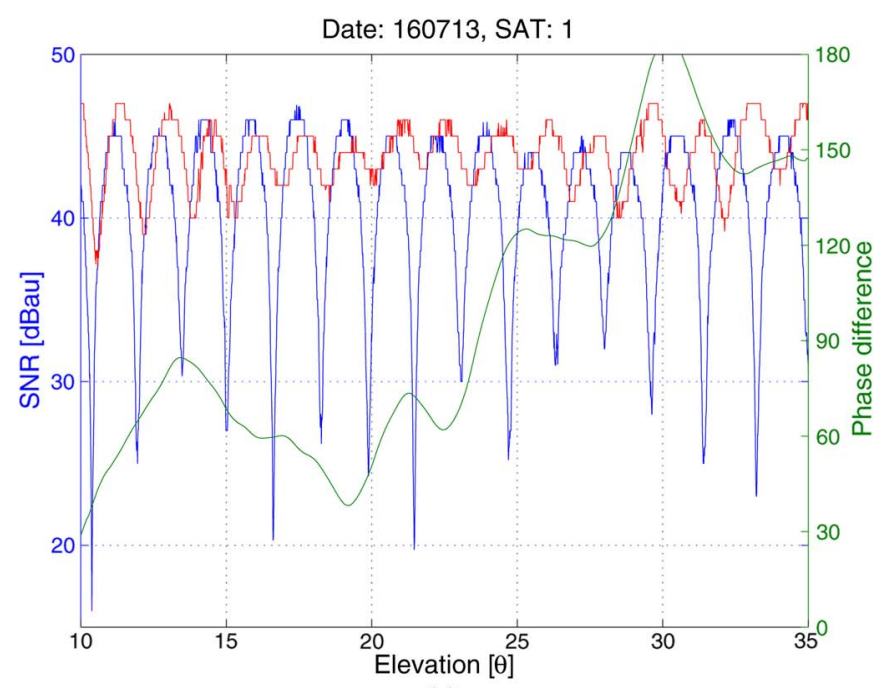

(a)

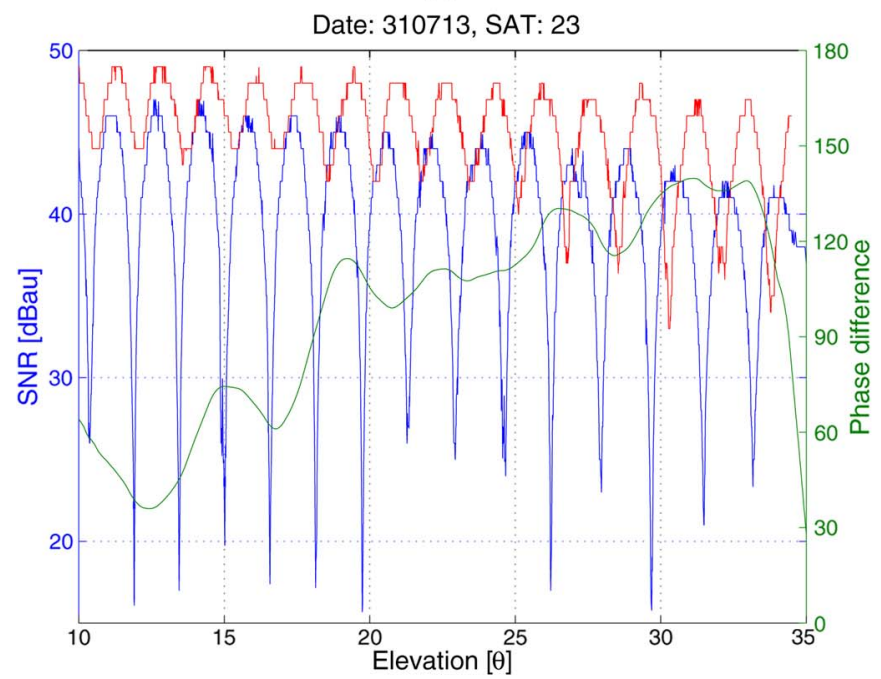

(b)

Fig. 5. IPT at V-Pol (red) and H-Pol (blue) and the phase difference (green) between them. (a) PRN 1 on July 16. (b) PRN 23 on July 31.

Two different data sets are shown in Fig. 5. As seen in Fig. 5(a), the identification of $\theta_{B}$ is not so straightforward when the frequency of the oscillations is not high enough, or what is the same, when there is a height limitation, since the lower the height, the lower the frequency of the oscillations. However, the $90^{\circ}$ phase difference position is clearly identified, and it 


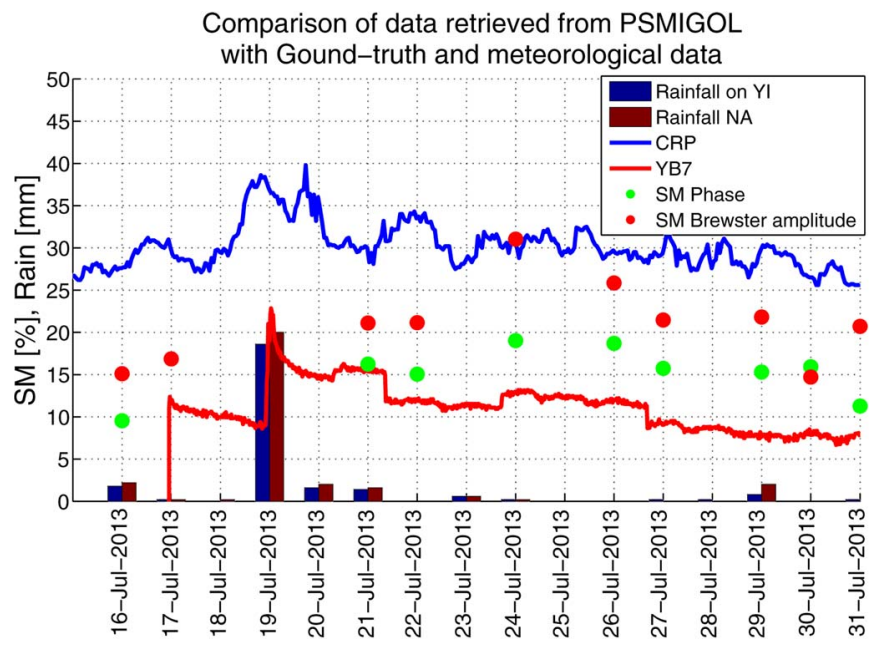

Fig. 6. Summary figure comparing with ground-truth retrievals. The blue and red bars represent rainfalls. (Blue line) Data from the CRP. (Red line) Data from the SDI-12 SM probe. (Green dots) Mean SM retrieved from phase measurements. (Red dots) Mean SM retrieved from Brewster angle position.

corresponds to $\theta_{B}$ value $\left(23^{\circ}\right)$. Getting this value as a reference (6\% SM), a SM map can be retrieved. The same effect occurs in Fig. 5(b), but now the amplitude of the oscillations at V-Pol is nearly the same for $\theta_{\text {elev }}$ in the range of $10^{\circ}-17^{\circ}$. However, using the phase difference observation, the $\theta_{B}$ position can be precisely detected $\left(18^{\circ}\right)$. Note that $\mathrm{SM}$ is not necessarily constant in the whole field, and this is the reason why in Fig. 5 the phase difference curves do not exactly follow the sharp shape shown in Fig. 3. However, the $90^{\circ}$ phase shift is a precise proxy of $\theta_{B}$.

To end this section, Fig. 6 compares the data series of the mean SM values retrieved using the phase retrieval algorithm with the Brewster angle position algorithm and external groundtruth SM data and the rainfall events in the period from the two closest meteorological stations. During the period analyzed, there was only one significant rain event on July 19. As it can be observed, the SM retrieved from the phase algorithm is more constant than the one retrieved with $\theta_{B}$. Then, the SM retrieved is generally in between the SDI-12 SM probe and the cosmic ray probe (CRP). This is due to the different penetration depths of both instruments. The SDI-12 SM probe measures the average SM of the terrain's first $5 \mathrm{~cm}$, whereas the CRP measures the area-average SM of a 200-m-diameter region around the probe with a penetration depth in between 10 and $25 \mathrm{~cm}$. The PSMIGOL reflectometer has a penetration depth in between 5 and $15 \mathrm{~cm}$ depending on SM conditions, which is in between the range of the SDI-12 probe and the CRP.

\section{CONCLUSION}

This letter has presented a new method to estimate more precisely the $\theta_{B}$ position. If $\theta_{B}$ is aimed to be precisely estimated, high-frequency oscillations of the IPT are required, which means installing instruments at heights typically higher than $8 \mathrm{~m}$, which is not always feasible. This results in much lower oscillation frequency, and consequently, it is more difficult to estimate the $\theta_{B}$ position by measuring the envelope of the IPT. The phase difference of the IPTs measured at H-Pol and V-Pol can be used to better estimate $\theta_{B}$, which results in an improvement in the SM estimation accuracy. Experimental results applying this technique have been shown in Section III, obtaining smoother variations than with the minimum amplitude detection algorithm.

Since the IPT relies on the coherent scattering of the reflected signals, the Rayleigh criterion for smooth surfaces must be satisfied for the reflecting surface.

\section{REFERENCES}

[1] H. J. Kramer, Observation of the Earth and Its Environment: Survey of Missions and Sensors. New York, NY, USA: Springer-Verlag, 1994.

[2] G. Krieger et al., "TanDEM-X: A satellite formation for high-resolution SAR interferometry," IEEE Trans. Geosci. Remote Sens., vol. 45, no. 11, pp. 3317-3341, Nov. 2007.

[3] J. Font et al., "SMOS: The challenging sea surface salinity measurement from space," Proc. IEEE, vol. 98, no. 5, pp. 649-665, May 2010.

[4] Y. Kerr et al., "The SMOS mission: New tool for monitoring key elements of the global water cycle," Proc. IEEE, vol. 98, no. 5, pp. 666-687, May 2010.

[5] D. Le Vine, G. S. E. Lagerloef, and S. Torrusio, "Aquarius and remote sensing of sea surface salinity from space," Proc. IEEE, vol. 98, no. 5, pp. 688-703, May 2010.

[6] S. J. and A. Komjathy, "GNSS reflectometry and remote sensing: New objectives and results," Adv. Space Res., vol. 46, pp. 111-117, 2010.

[7] J.-C. Auber, A. Bibaut, and J.-M. Rigal, "Characterization of multipath on land and sea at GPS frequencies," in Proc. 7th Int. Meet. Satellite Division ION GPS, Sep. 1994, pp. 1155-1171.

[8] M. Martín-Neira, "A passive reflectometry and interferometry system (PARIS): Application to ocean altimetry," ESA J., vol. 17, pp. 331-355, 1993.

[9] E. Cardellach, "GNSS-R ground-based and airborne campaigns for ocean, land, ice, snow techniques: Applications to the GOLD-RTR data sets," Radio Sci., vol. 46, no. 6, pp. RS0C04-1-RS0C04-16, Dec. 2011.

[10] V. Zavorotny et al., "Seasonal polarimetric measurements of soil moisture using tower-based GPS bistatic radar," in Proc. IEEE IGARSS, 2003, vol. 2, pp. 781-783.

[11] D. Masters, P. Axelrad, and S. Katzberg, "Initial results of land-reflected GPS bistatic radar measurements in SMEX02," Remote Sens. Environ., vol. 92 , no. 4, pp. 507-520, Sep. 2004.

[12] A. Kavak, W. Vogel, and G. Xu, "Using GPS to measure ground complex permittivity," Electron. Lett., vol. 34, no. 3, pp. 254-255, Feb. 1998.

[13] K. Larson et al., "Using GPS multipath to measure soil moisture fluctuations: Initial results," GPS Solutions, vol. 12, no. 3, pp. 173-177, Jul. 2008, [Online]. Available: http://dx.doi.org/10.1007/s10291-007-0076-6

[14] V. Zavorotny et al., "A physical model for GPS multipath caused by land reflections: Toward bare soil moisture retrievals," IEEE J. Sel. Topics Applied Earth Observ. Remote Sens., vol. 3, no. 1, pp. 100-110, Mar. 2010.

[15] C. Chew, E. Small, K. Larson, and V. Zavorotny, "Effects of near-surface soil moisture on GPS SNR data: Development of a retrieval algorithm for soil moisture," IEEE Trans. Geosci. Remote Sens., vol. 52, no. 1, pp. 537-543, Jan. 2014.

[16] N. Rodriguez-Alvarez et al., "Soil moisture retrieval using GNSS-R techniques: Experimental results over a bare soil field," IEEE Trans. Geosci. Remote Sens., vol. 47, no. 11, pp. 3616-3624, Nov. 2009.

[17] N. Rodriguez-Alvarez et al., "Land geophysical parameters retrieval using the interference pattern GNSS-R technique," IEEE Trans. Geosci. Remote Sens., vol. 49, no. 1, pp. 71-84, Jan. 2011.

[18] A. Egido et al., "Global navigation satellite systems reflectometry as a remote sensing tool for agriculture," Remote Sens., vol. 4, no. 8, pp. 2356 2372, Aug. 2012.

[19] P. Beckmann and A. Spizzichino, The Scattering of Electromagnetic Waves From Rough Surfaces. New York, NY, USA: Pergamon, 1993.

[20] G. Lérondel and R. Romestain, "Fresnel coefficients of a rough interface," Appl. Phys. Lett., vol. 74, no. 19, pp. 2740-2742, May 1999.

[21] J. Wang and T. Schmugge, "An empirical model for the complex dielectric permittivity of soils as a function of water content," IEEE Trans. Geosci. Remote Sens., vol. GE-18, no. 4, pp. 288-295, Oct. 1980.

[22] SDI-12 SM Probe. [Online]. Available: http://www.oznet.org.au/SDI-12. html 